\title{
Manipulating superconducting phases via current-driven magnetic states in rare-earth-doped $\mathrm{CaFe}_{2} \mathrm{As}_{2}$
}

\author{
Soon-Gil Jung $\mathbb{1}^{1}$, Soohyeon Shin ${ }^{1}$, Harim Jang ${ }^{1}$, Won Nam Kang², Jeong Hwan Han³, Akinori Mine,
} Tsuyoshi Tamegai ${ }^{4}$ and Tuson Park ${ }^{1}$

\begin{abstract}
Inhomogeneous superconductivity in rare-earth (RE)-doped $\mathrm{CaFe}_{2} \mathrm{As}_{2}$ (Ca122) compounds leads to a novel state of matter in which the superconducting and magnetic states can be simultaneously controlled by using an electric current (). Both La- and Ce-doped Ca122 single crystals show a very broad superconducting transition width $\left(\Delta T_{c}\right)$ due to their non-bulk nature. Surprisingly, $\Delta T_{\mathrm{c}}$ becomes sharper or broader after an electric current larger than a threshold value $\left(I_{t}\right)$ is applied, with a concomitant change in the normal-state magnetism. The sharpened (broadened) $\Delta T_{\mathrm{c}}$ is accompanied by a decrease (an increase) in the amplitude of the ferromagnetic signals. The sensitive changes in the superconductivity and magnetism that occur when an external current is applied are related to the inhomogeneous electronic states that originate from the Fe magnetic state and/or self-organized superconducting/magnetic composites in Ca122 compounds. These discoveries shed new light on the role of Fe in Fe-based superconductors and will provide new ideas for the design of novel superconducting devices.
\end{abstract}

\section{Introduction}

The magnetic state of Fe coupled with interlayer As-As bonding has been intensively investigated as a crucial source to mediate Cooper pairs in Fe-based superconductors $(\mathrm{FeSCs})^{1-5}$. The $\mathrm{CaFe}_{2} \mathrm{As}_{2}$ (Ca122) compound is an ideal candidate for studying the influence of the $\mathrm{Fe}$ magnetic state on FeSCs due to its extremely sensitive lattice parameters, which affect the Fe magnetic state $^{1,2,4-7}$. Therefore, recent results for the Ca122 system have mostly focused on the relation between the $\mathrm{Fe}$ magnetic state and superconductivity because the interplay between magnetism and superconductivity is a key to understanding the mechanisms at play in FeSCs,

\footnotetext{
Correspondence: Tuson Park (tp8701@skku.edu)

${ }^{1}$ Center for Quantum Materials and Superconductivity (CQMS), Department of Physics, Sungkyunkwan University, Suwon 16419, Republic of Korea

${ }^{2}$ Department of Physics, Sungkyunkwan University, Suwon 16419, Republic of Korea

Full list of author information is available at the end of the article
}

as well as other unconventional superconductors, such as high- $T_{\mathrm{c}}$ cuprates and heavy fermion superconductors ${ }^{8,9}$.

Recently, a superconducting transition temperature $\left(T_{\mathrm{c}}\right)$ as high as $49 \mathrm{~K}$ was reported in rare-earth (RE: $\mathrm{La}, \mathrm{Ce}, \mathrm{Pr}$, $\mathrm{Nd}$ )-doped Ca122 compounds ${ }^{10-12}$. However, the nature of the high $-T_{\mathrm{c}}$ is unclear due to the small volume fractions of the high- $T_{\mathrm{c}}$ phases and their inhomogeneities. High- $T_{\mathrm{c}}$ regions have been reported to be nucleated around doped $R E$ clusters ${ }^{11,13}$. The results of scanning tunneling microscopy (STM) measurements for Pr-doped Ca122 indicate that the high $-T_{\mathrm{c}}$ regions around the Pr clusters are surrounded by weak- or non-SC regions ${ }^{11}$. Therefore, the supercurrent will mainly percolate along weak-SC or non-SC regions, leading to another $\mathrm{SC}$ transition at temperatures of approximately $10-20 \mathrm{~K}$ in $R E$-doped Ca122 compounds ${ }^{11,12,14}$.

The inhomogeneities or phase separations in a material often complicate the investigation of its intrinsic physical properties due to secondary effects. On the other hand, such complexity may bring about unexpected results,

\section{(c) The Author(s) 2018}

(c) (i) Open Access This article is licensed under a Creative Commons Attribution 4.0 International License, which permits use, sharing, adaptation, distribution and reproduction cc) in any medium or format, as long as you give appropriate credit to the original author(s) and the source, provide a link to the Creative Commons license, and indicate if changes were made. The images or other third party material in this article are included in the article's Creative Commons license, unless indicated otherwise in a credit line to the material. If material is not included in the article's Creative Commons license and your intended use is not permitted by statutory regulation or exceeds the permitted use, you will need to obtain permission directly from the copyright holder. To view a copy of this license, visit http://creativecommons.org/licenses/by/4.0/. 

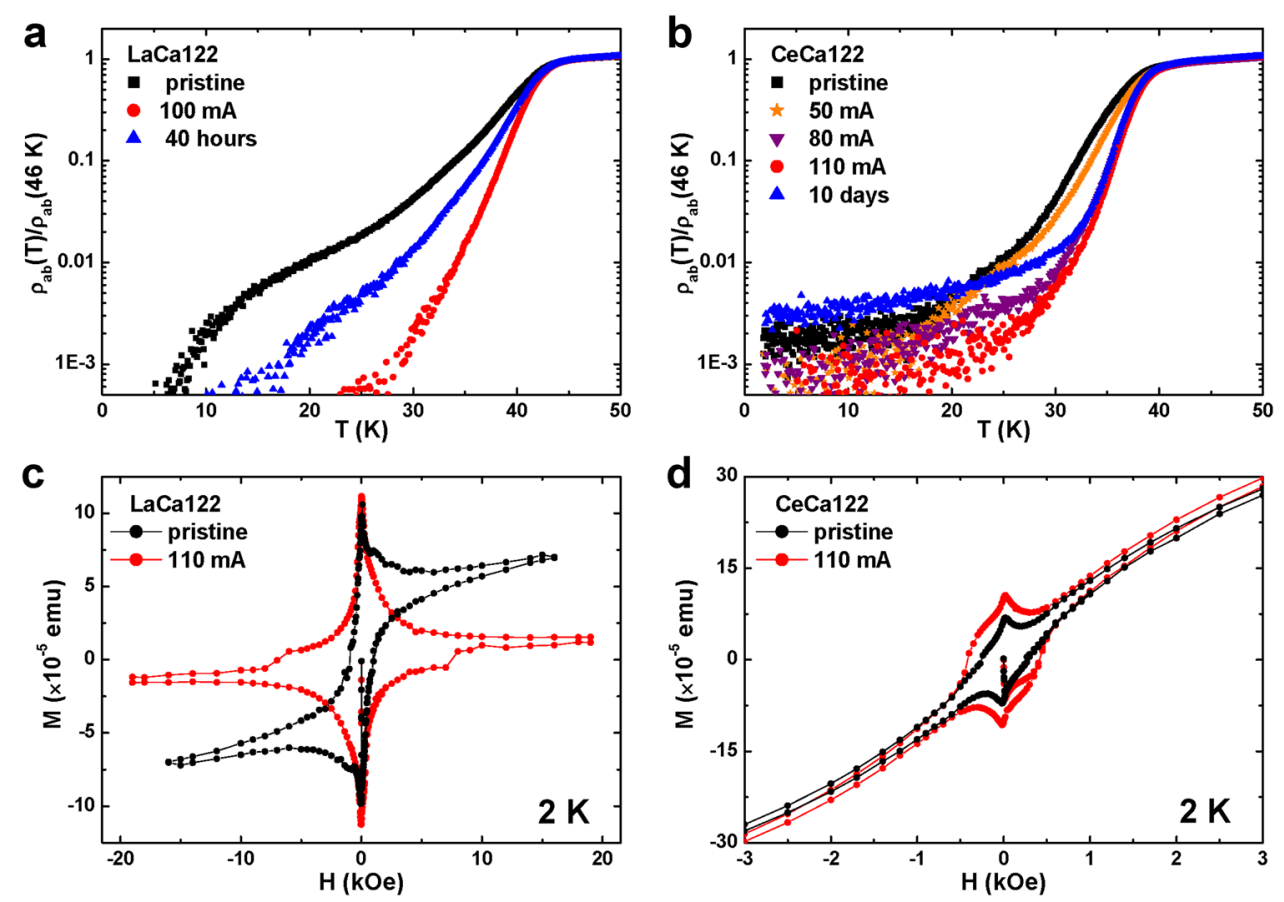

Fig. 1 Effects of an electric current on rare-earth-doped $\mathrm{CaFe}_{2} \mathbf{A s}_{2}$ compounds. Temperature dependence of the in-plane resistivity $\left(\rho_{\mathrm{ab}}\right)$ for a La-doped $\mathrm{CaFe}_{2} \mathrm{As}_{2}$ (LaCa122) and $\mathbf{b}$ Ce-doped $\mathrm{CaFe}_{2} \mathrm{As}_{2}$ (CeCa122), where $\rho_{\mathrm{ab}}$ is normalized to the value at $46 \mathrm{~K}$. For LaCa122, the zero-resistivity temperature is much improved after application of a large current of $100 \mathrm{~mA}$, and aging effects clearly seen in the SC transition curves. CeCa122 also shows a behavior similar to that of LaCa122 following application of an electric current. Magnetization hysteresis $(M-H)$ loops at $2 \mathrm{~K}$ for $\mathbf{c}$ LaCa122 and $\mathbf{d}$ CeCa122. Interestingly, for both samples, the $M-H$ curves are also changed when a current is applied, indicating that the current-driven magnetic state affects the SC state for La- and Ce-doped Ca122. The weak-SC signals in the $M-H$ loops for CeCa122 are most likely due to the strong Ce $4 f$ magnetic moment

including disorder-induced superconductivity ${ }^{15,16}$. Complex phases in $R E$-doped Ca122 produce unpredicted results, such as a magnetic state that can be manipulated by using an electric current, which consequently affects the SC state. A superconducting current can be a good source for controlling the magnetic moment of nanoscale magnetic clusters as it is large enough to switch the magnetization. The effective magnetic field generated by the superconducting current affects the magnetic impurities/clusters; thus, the magnetic state of magnetic clusters with a large anisotropy can be manipulated ${ }^{17,18}$.

In this study, we report on manipulating the superconductivity of La- and Ce-doped Ca122 crystals by using the current-driven magnetic state. The SC percolation path, which is mainly composed of the low- $T_{\mathrm{c}}$ phase, is greatly modified, as is the magnetic state, when a current larger than a threshold value $\left(I_{\mathrm{t}}\right)$ is applied, but the high$T_{\mathrm{c}}$ phase is not significantly changed. In addition, both the transport and magnetization measurements show aging effects. These novel behaviors can be attributed to the sensitive Fe magnetic state or to self-assembled superconducting/magnetic composites inside the crystals.

\section{Materials and methods}

\section{Synthesis and characterization of rare-earth-doped}

\section{$\mathrm{CaFe}_{2} \mathrm{As}_{2}$ single crystals}

Undoped and rare-earth (RE)-doped $\mathrm{CaFe}_{2} \mathrm{As}_{2}$ single crystals were grown by using the FeAs self-flux method $^{6,19}$; these crystals were easily cleaved into platelike shapes with typical sizes of $4 \times 2 \times 0.25 \mathrm{~mm}^{3}$. Binary FeAs was synthesized by reacting Fe powder (99.998\%, Alfa Aesar, Ward Hill, MA, USA) with an As lump (99.999\%, Alfa Aesar) in a quartz tube that was heated at $500{ }^{\circ} \mathrm{C}$ for $10 \mathrm{~h}$ and then at $700^{\circ} \mathrm{C}$ for $10 \mathrm{~h}$ in a box-type furnace. The elements $\mathrm{Ca}$ (granules 99.5\%, Alfa Aesar), Ce, and La (both rods 99.9\%, Alfa Aesar) as well as the FeAs precursor were placed into an alumina crucible at a ratio of $(\mathrm{Ca}, \mathrm{La}$, or $\mathrm{Ce})$ : $\mathrm{FeAs}=1: 4$ and then sealed in an evacuated quartz tube. The ampoules were heated at $1180^{\circ} \mathrm{C}$ for $24 \mathrm{~h}$ before being slowly cooled to $960{ }^{\circ} \mathrm{C}$ at a rate of $4{ }^{\circ} \mathrm{C} / \mathrm{h}$. The heater was then turned off at that temperature. The composition ratio of the synthesized $\mathrm{CaFe}_{2} \mathrm{As}_{2}$ compounds was investigated by using energy dispersive spectroscopy (EDS). 
Measurements of electronic transport and magnetization

The electrical resistivity of the superconducting transition was measured by using the standard four-probe method with an applied current of $1 \mathrm{~mA}$ for all samples, with the measurements carried out in a physical property measurement system (PPMS 9T, Quantum Design). Small, pure, and $R E$-doped $\mathrm{CaFe}_{2} \mathrm{As}_{2}$ pieces that cleaved from the large samples, with a typical size of $1.2-2.7 \times$ $0.3 \times 0.1 \mathrm{~mm}^{3}$, were used to investigate the effects of the electric current on the materials. Pt wires and Epotek H20E silver epoxy were used to make good electrical contacts. Large electrical currents, up to $110 \mathrm{~mA}$, were generated by using an Advantest R6142 unit under cryogenic conditions (5 and $10 \mathrm{~K}$ ). The magnetization hysteresis $(M-H)$ loops and temperature dependence of the magnetization $(M)$ were measured by using a magnetic property measurement system (MPMS 5T, Quantum Design). To observe the effects of aging, we placed the samples inside desiccators at room temperature.

\section{Results}

Figure 1 shows representative results for the superconducting (SC) and magnetic states in La- and Ce-doped
Ca122 crystals that were tuned by application of an electric current. The SC transition widths $\left(\Delta T_{\mathrm{c}}\right)$ for both La- and Ce-doped Ca122 become sharper after a current larger than a threshold value $\left(I>I_{\mathrm{t}}\right)$ is applied to the samples, while the temperature for the onset of superconductivity $\left(T_{\mathrm{c}, \text { onset }}\right)$ does not change significantly, as shown in Fig. 1a, b. Simultaneously, the SC magnetization hysteresis $(M-H)$ loop is prominently modified, which is accompanied by a large suppression in the ferromagnetic (FM) signal for La-doped Ca122, as shown in Fig. 1c. On the other hand, the slightly improved SC $M-H$ loop observed for Ce-doped Ca122 can be ascribed to the relatively large magnetic moment of Ce at low temperatures, as shown in Fig. 1d (see also Figure $\mathrm{S} 1$ in $\mathrm{SI})^{13}$. The exact origin of the FM phase in $R E$ doped Ca122 compounds is still unclear, but the defects at the Fe/As sites, such as As deficiency or Fe excess, have been suggested to be a source of the FM phase in $\mathrm{FeSCs}^{13,20-22}$.

Aging effects are also observed in the temperature dependence of the in-plane electrical resistivity $\left(\rho_{\mathrm{ab}}\right)$, as presented in Fig. 1a. In La-doped Ca122, $\Delta T_{\mathrm{c}}$ is sharpened by applying a current of $100 \mathrm{~mA}$, but it becomes broader a

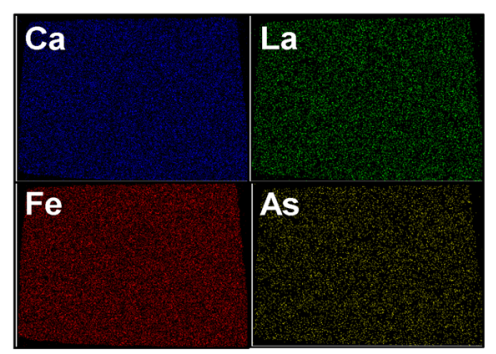

C

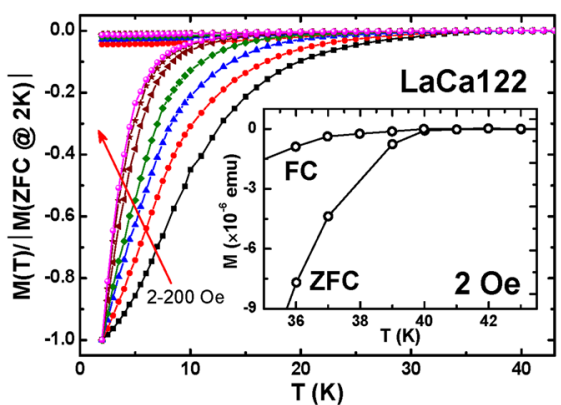

b

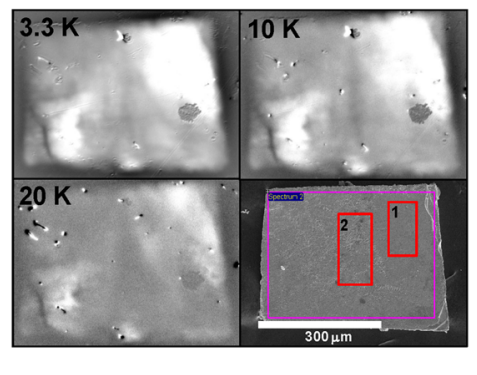

d

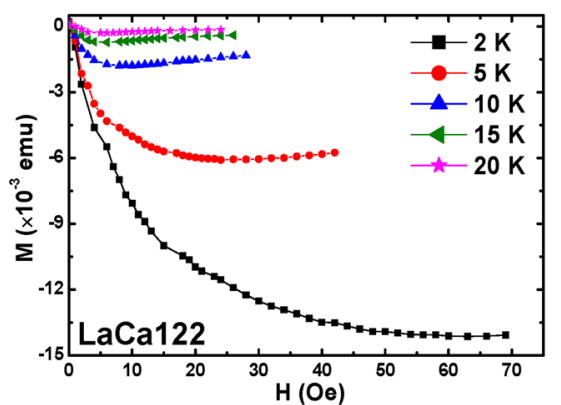

Fig. 2 EDS elemental mapping and superconducting magnetization properties for La-doped $\mathrm{CaFe}_{2} \mathrm{As}_{\mathbf{2}}$. a The EDS elemental mapping for LaCa122 shows that all of the elements in the crystal are homogeneously distributed. $\mathbf{b}$ However, the magneto-optical (MO) images show localized strong-SC regions. The $\mathrm{MO}$ images at 3.3, 10, and $20 \mathrm{~K}$ are measured in the remanent states, and the bright regions indicate strong-SC regions, where the high- $T_{c}$ phase is dominant. The EDS results in regions 1 and 2 are not different despite the presence of very different SC states, implying that nanoscale inhomogeneity is important. c Zero-field-cooled (ZFC) and field-cooled (FC) dc magnetization (M) data for applied magnetic fields of 2, 5, $10,20,50,100$, and 200 Oe. Here, M(T) is normalized to the ZFC M value at $2 \mathrm{~K}$. As shown in the inset, a clear separation between the ZFC and FC data at $2 \mathrm{Oe}$ is observed at temperatures below $40 \mathrm{~K}$, but the SC volume fraction at high temperatures is easily suppressed by small magnetic fields. $\mathbf{d}$ The magnetic field dependence of $M$ at temperatures of $2,5,10,15$, and $20 \mathrm{~K}$. The rapid suppression of $M(H)$ at high temperatures implies a small volume fraction for the high- $T_{c}$ phase in LaCa122 
after $40 \mathrm{~h}$. A similar behavior can also be observed in Ce-doped Ca122, as shown in Fig. 1b. These unexpected features in RE-doped Ca122 are more prominent in Ladoped Ca122 than Ce-doped Ca122. Hence, we focus on La-doped Ca122 to investigate the origin of the changes in superconductivity and magnetism induced by applying a current in RE-doped Ca122 compounds.

Synthesized La-doped $\mathrm{CaFe}_{2} \mathrm{As}_{2}$ single crystals were easily cleaved into plate-like shapes with a typical size of $4 \times 2 \times 0.25 \mathrm{~mm}^{3}$. Homogeneous distributions for all of the elements in the crystals were confirmed by using energy dispersive spectroscopy (EDS) elemental mapping, as shown by the results presented in Fig. 2a. Nanoscale inhomogeneities have been suggested to be important for understanding the origin of the high- $T_{\mathrm{c}}$ phase in $R E$ doped Ca122 superconductors ${ }^{11,13}$. Figure $2 \mathrm{~b}$ shows a magneto-optical (MO) image in the remanent state measured at $3.3,10$, and $20 \mathrm{~K}$. The bright regions indicate strong-SC phases, and the dark regions show weak- or non-SC regions, indicating that the high- $T_{\mathrm{c}}$ phase is localized and rapidly suppressed with increasing temperature (see also Figure S2 in SI). Inhomogeneously distributed SC areas cannot be clearly distinguished by using EDS elemental mapping, which is particularly true for the La concentrations. The La concentrations of the rectangular region that has a magenta color and for regions 1 and 2 in Fig. $2 \mathrm{~b}$ are $18.6 \%$ and $20.4 \%$, respectively.

The strong temperature dependence and small volume fraction of the high- $T_{\mathrm{c}}$ phase are also observed in the magnetization $(M)$ characteristics for the large size Ladoped Ca122 crystal $\left(4.19 \times 2.09 \times 0.25 \mathrm{~mm}^{3}\right)$. The zerofield-cooled (ZFC) and field-cooled (FC) dc $M$ for Ladoped Ca122 in magnetic fields of 2, 5, 10, 20, 50, 100, and 200 Oe are shown in Fig. 2c. Here, $M(T)$ is normalized to the ZFC $M$ value at $2 \mathrm{~K}$ for comparison. The ZFC $M(T)$ shows a rapid suppression even at small magnetic fields, although an irreversible line between the ZFC and FC data measured at $2 \mathrm{Oe}$ is clearly observed at $40 \mathrm{~K}$, as shown in the inset of Fig. 2c. Figure 2d shows the $M-H$ curves at low fields for several representative temperatures: $2,5,10$, 15 , and $20 \mathrm{~K}$. The large reduction in the Meissner signal caused by small magnetic fields also indicates a small volume fraction for the high- $T_{\mathrm{c}}$ phase. The diamagnetic signals in the Meissner state are significantly suppressed at temperatures higher than $10 \mathrm{~K}$, which corresponds to

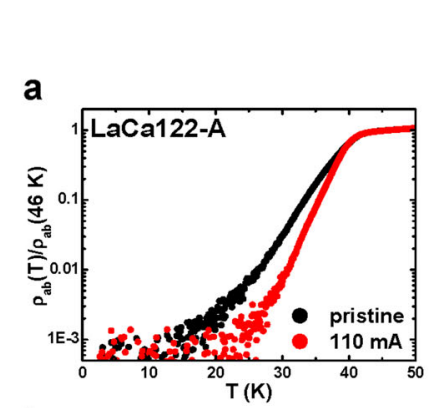

b

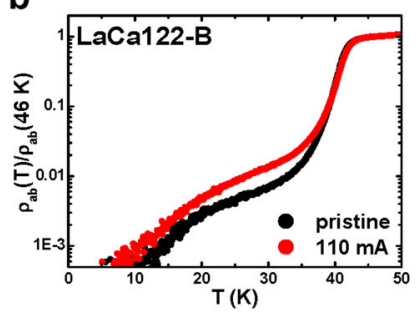

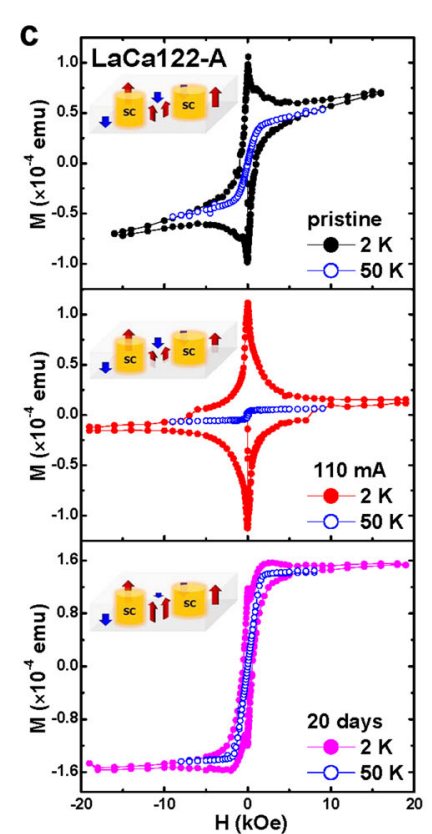

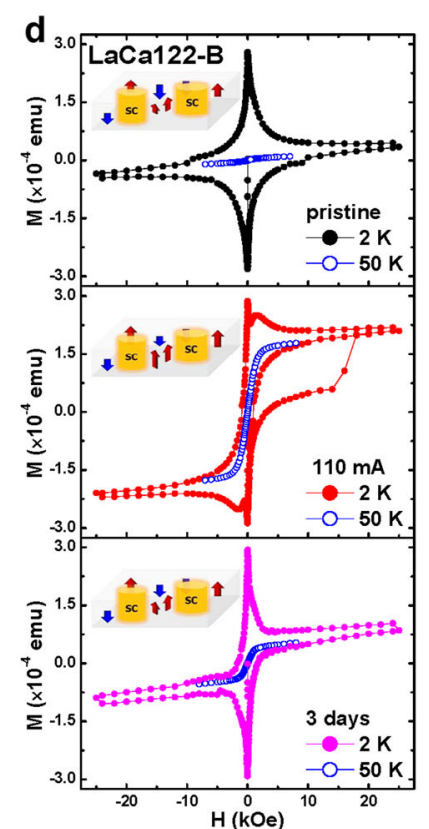

Fig. 3 Manipulation of the superconducting states for two pieces of La-doped $\mathrm{CaFe}_{2} \mathrm{As}_{\mathbf{2}}$ by using an electric current. Temperature dependence of the normalized in-plane resistivity $\left(\rho_{\mathrm{ab}}\right)$ for $\mathbf{a}$ LaCa122-A and $\mathbf{b}$ LaCa122-B, where the resistivity is normalized to its value at $46 \mathrm{~K}$. The SC transition width for LaCa122-A becomes narrower after application of a current, whereas LaCa122-B shows a broader SC transition compared to the pristine state. $\mathrm{M}-\mathrm{H}$ loops at 2 and $50 \mathrm{~K}$ for $\mathbf{c} \mathrm{LaCa} 122-\mathrm{A}$ and $\mathbf{d} \mathrm{LaCa} 122-\mathrm{B}$, indicating that the normal-state magnetism (50 K), which is Changed by the electric current, strongly affects the shape of the SC $M-H$ loops. In addition, the changes in the SC transition in the $\rho_{\mathrm{ab}}(\mathrm{T})$ curves correspond to the modified $M-H$ loops. For instance, suppression of the FM signal when a current is applied in LaCa122-A may indicate improved SC percolation paths because ferromagnetism is detrimental to superconductivity. The pictures in the insets of (c, d) simply describe the high- $T_{c}$ regions and magnetic domains in each case based on the $\mathrm{MO}$ images and the $M-H$ curves at $50 \mathrm{~K}$. The red and blue arrows represent the "up" and "down" direction of the magnetization, respectively, while the length of the arrow corresponds to the magnitude of the magnetic domain 
the MO images shown in Fig. 2b. The large suppression of the SC diamagnetic signal by small perturbations, such as temperature and magnetic field perturbations, is consistent with a small SC volume fraction and/or with isolated SC domains with small sizes comparable to the penetration depth $(\lambda)^{10,23}$.

We selected two types of small La-doped Ca122 pieces, LaCa122-A and LaCa122-B, which have SC states that can be tuned by using the current-driven magnetic state (see Figure $\mathrm{S} 3$ in SI). The La concentration estimated by EDS is $17.9 \%$ and $18.1 \%$ for $\mathrm{LaCa} 122-\mathrm{A}$ and $\mathrm{LaCa} 22-\mathrm{B}$, respectively. Figure 3a, b plots the values for $\rho_{\mathrm{ab}}(T)$ normalized to the value at $46 \mathrm{~K}, \rho_{\mathrm{ab}}(T) / \rho_{\mathrm{ab}}(46 \mathrm{~K})$, for LaCa122-A and $-\mathrm{B}$, respectively, on a semi-logarithmic scale. When a large electric current $(110 \mathrm{~mA})$ is applied, the SC transition for LaCa122-A becomes sharper, while it is broadened for LaCa122-B. Multiple SC phases with a low and high- $T_{\mathrm{c}}$ were clearly observed in LaCa122-B, which has been widely reported in $R E$-doped $\mathrm{CaFe}_{2} \mathrm{As}_{2}$ (see also Figure S4 in SI) ${ }^{10-14,24}$.

Figure 3c, d shows the $M-H$ loops for LaCa122-A and -B at $2 \mathrm{~K}\left(T<T_{\mathrm{c}}\right)$ and $50 \mathrm{~K}\left(T>T_{\mathrm{c}}\right)$, where the top, middle, and bottom panels show the results for pristine crystals, crystals treated with a current of $110 \mathrm{~mA}$, and aged single crystals. The pristine states for LaCa122-A and -B are different. LaCa122-A has a stronger FM signal compared to $\mathrm{LaCa} 122-\mathrm{B}$, and the shape of the $M-H$ curves at $2 \mathrm{~K}$ is reversed after application of a large current of $110 \mathrm{~mA}$. Interestingly, the $M-H$ loops also show aging effects, but they do not return to their initial states even after a relatively long time (see Figure S5 in SI). Similarly, $M-H$ curves have been reported in ferromagnet/superconductor systems, such as $\mathrm{Co} / \mathrm{Nb}$, with the shape of the $M-H$ curve controlled by the thickness of the SC or FM layer ${ }^{25,26}$. When the shapes of the $M-H$ curves at $2 \mathrm{~K}$ for LaCa122-A are considered, the states of pristine crystals, crystals treated with a current of $110 \mathrm{~mA}$, and crystals aged for 20 days are found to contain a mixed phase of SC and FM, dominant SC state, and dominant FM phase, respectively ${ }^{25}$.

\section{Discussion}

Current annealing has been used to rearrange the magnetic domains in Co-based amorphous ribbons through short-time heat treatment via Joule heating ${ }^{27,28}$. In the present study, the simultaneous changes in the SC and magnetic states due to the applied electric current are not a result of thermal annealing because aging effects are
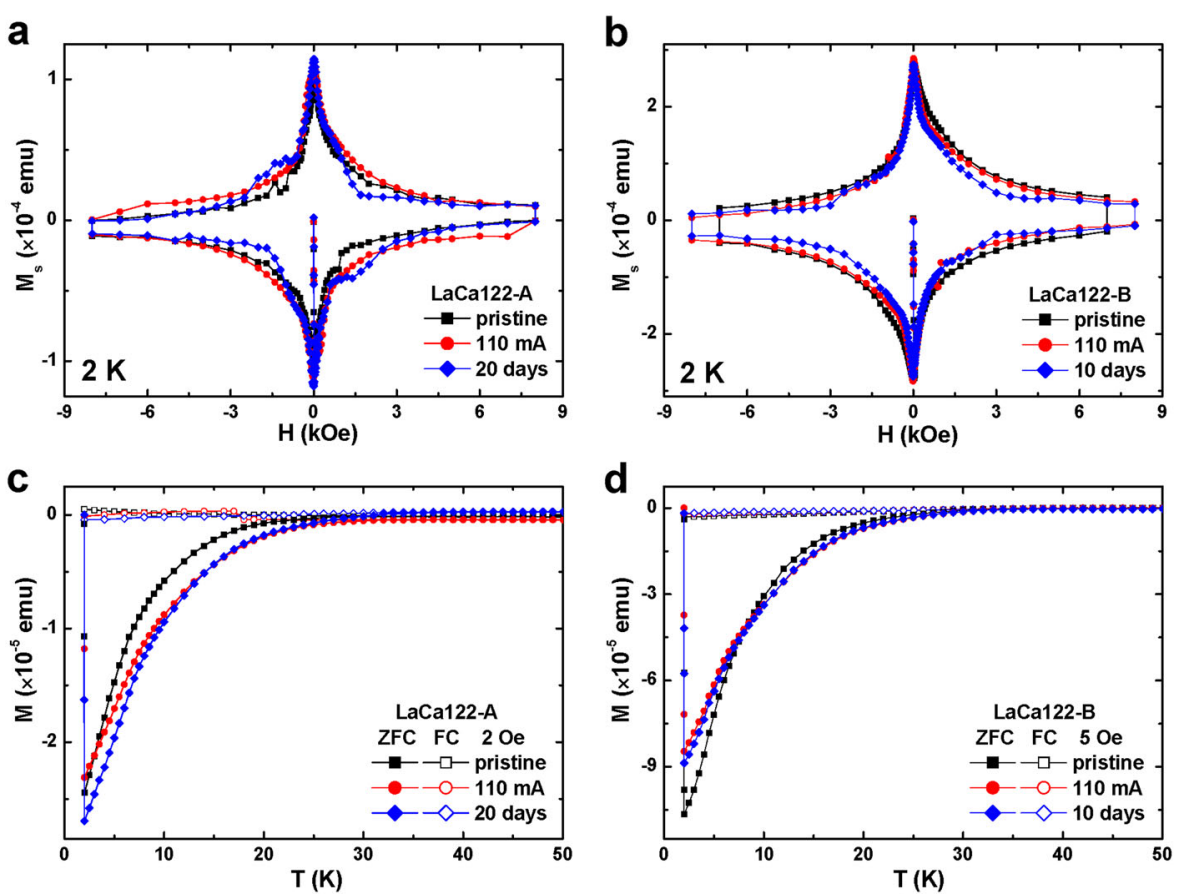

Fig. 4 Magnetization curves for the superconducting phase of La-doped $\mathrm{CaFe}_{2} \mathrm{As}_{2} \cdot M_{\mathrm{s}}-\mathrm{H}$ loops when pure SC phases are considered for a LaCa122-A and $\mathbf{b}$ LaCa122-B, respectively, where $M_{s}$ is the magnetization of the pure SC phase. Because the $M-H$ loops at 2 and $50 \mathrm{~K}$ are superimposed as the FM background, as shown in Fig. $3 c$, d, the $M_{s}-H$ loops can simply be calculated by using the relation $M_{s}(2 \mathrm{~K}, H)=M(2 \mathrm{~K}, H)-$ $M(50 \mathrm{~K}, H)$. The temperature dependence of the magnetization $(M-T)$ for $\mathbf{c}$ LaCa122-A and $\mathbf{d}$ LaCa122-B shows that the SC volume fraction barely changes with current and time. Zero-field cooling starts from zero gauss at $2 \mathrm{~K}$ for both samples, with the negligible $M$ values at zero gauss (remanent fields) for all cases, indicating that the $M-T$ results are acceptable 
observed and the current was applied under cryogenic conditions.

Magnetic states tuned by using the electric current and time are clearly seen for both LaCa122-A and -B, as shown in Fig. 3c, d, respectively. Pristine LaCa122-A is in a mixed phase of superconductivity and ferromagnetism. When a larger current of $110 \mathrm{~mA}$ is applied, however, the FM signal is considerably suppressed; consequently, a typical SC $M-H$ curve appears. In striking contrast, current-treated $\mathrm{LaCa} 122-\mathrm{A}$ recovers the large FM signal after storage for 20 days. On the other hand, LaCa122-B with a small FM signal in the pristine state shows the opposite behavior. When a current of $110 \mathrm{~mA}$ is applied, the FM signal increases significantly, followed by a rapid decrease over a period of 3 days, after which it hardly changes over the next 47 days (50-day observation period) (see Figures $\mathrm{S} 5$ and $\mathrm{S} 6$ in $\mathrm{SI}$ ).

The $M-H$ loops for all cases measured at 2 and $50 \mathrm{~K}$ are superimposed on top of a linear background, and $M_{\mathrm{s}}-H$ loops for the pure SC phase can be obtained, as shown in Fig. 4a, b, where $M_{\mathrm{s}}(2 \mathrm{~K}, H)=M(2 \mathrm{~K}, H)-$ $M(50 \mathrm{~K}, H)$. The $M_{\mathrm{s}}-H$ loops for both LaCa122-A and -B present no significant changes in the high- $T_{\mathrm{c}}$ phase with changing current and/or time, implying that the current-driven magnetic state and simultaneous change in the SC state mostly emerge in weak- or non-SC regions. The temperature dependence of the magnetization $(M-T)$ also presents a rare change in the $\mathrm{SC}$ volume fraction following application of a current, as shown in Fig. 4c, d. Measurements for zero-field cooling (ZFC) were started from " 0 " gauss at $2 \mathrm{~K}$; the negligible $M$ values at " 0 " gauss (remanent fields) for all cases indicate that the $M-T$ results are reliable. The lack of change in the ZFC and $M_{\mathrm{s}}-H$ data suggests that the percolation paths surrounding the high $-T_{\mathrm{c}}$ phases are mainly affected by the applied electric current.

In order to understand the different $M-H$ behaviors for LaCa122-A and -B, we considered the simple pictorials, taking into account the current and relaxation time, as described in the insets of Fig. 3c, d. First, the size of the magnetic signals for La-doped Ca122 compounds is changed by applying an electric current, but the magnetic states return to their original states because the currentdriven magnetic states are unstable. However, the observation that the $M-H$ curves for the final states are not exactly the same as those for the initial states is probably strongly related to the relaxation time. For example, the observation that the FM signal is enhanced after 20 days for LaCa122-A in comparison with the initial FM signal is closely related to the relatively long relaxation time because the surroundings can be greatly influenced by the FM state during such a long relaxation time.

Current-driven magnetization switching is a central issue in the field of spintronics. We considered the threshold current density $\left(J_{\mathrm{t}} \sim 10^{7}-10^{8} \mathrm{~A} / \mathrm{cm}^{2}\right)$ for magnetization switching in FM/SC or FM/AFM metallic composites $^{29,30}$. In this study, large changes in the $\Delta T_{\mathrm{c}}$ and $M-H$ curves were mostly observed at applied currents between 50 and $80 \mathrm{~mA}$, as shown in Fig. $1 \mathrm{~b}$ (see also Figure S7 in SI). If the percolation path has the shape of a long wire and $J_{\mathrm{t}}=10^{7}-10^{8} \mathrm{~A} / \mathrm{cm}^{2}$, the estimated average radius $r_{\text {avg }}$ is a few hundred nanometers (see also Figure $\mathrm{S} 8$ in $\mathrm{SI}$ ), which is close to the typical penetration depth $\left(\lambda_{\mathrm{ab}}\right)$ for $\mathrm{FeSCs}^{31}$. Recently, the possibility of manipulating magnetic moments by using superconducting currents was suggested theoretically, considering the interplay between the spin-orbit interaction and a large transport current ${ }^{17,18}$. Persistent currents can be induced to circulate in FM impurities/islands with radii $R<\xi$ (SC coherence length) on an $s$-wave 2D SC layer with large spin-orbit coupling (SOC), thereby generating large magnetic anisotropy. In addition, during electrical current flow in a superconductor, an effective magnetic field is generated, which acts on the FM impurities/ islands; consequently, the orientations of the magnetic moments of the FM impurities/islands can be manipulated ${ }^{17,18}$.

The mechanism of the superconductivity in the Ca122 compounds is still unclear, but numerous results have suggested that an adequate Fe magnetic moment is crucial to mediating Cooper pairs in the compounds ${ }^{1,2,4}$. The interface superconductivity that emerges in undoped Ca122 has been reported to originate from the interface between the collapsed tetragonal (cT) and orthorhombic (O) phases or between the AFM antiphase domain boundaries $^{32,33}$. For undoped and Co-doped Ca122, nuclear magnetic resonance (NMR) results suggest that FM fluctuations compete with superconductivity but can coexist with a stripe-type AFM phase ${ }^{34}$. The high$T_{\mathrm{c}}$ phase in the $R E$-doped Ca122 is considered to be due to the interface associated with $R E$ dopants ${ }^{13,22,35,36}$. Zhou et al. recently reported evidence for the interfacial nature of high- $T_{\mathrm{c}}$ superconductivity, where the interface is located between the pristine and reconstructed FeAs layers near $R E$ dopants ${ }^{36}$. These complexities lead to unexpected results and allow manipulation of the SC and magnetic states by using an electric current, providing new insight into the role of $\mathrm{Fe}$ in Fe-based superconductors. We note that the switching of magnetism and superconductivity has also been reported by controlling the magnetic state of the $\mathrm{Fe}$ layer via the spin-polarized current in a FeSC $\mathrm{Sr}_{2} \mathrm{VO}_{3} \mathrm{FeAs}$ single crystal $^{21}$.

\section{Conclusion}

We discovered that the current-driven magnetic state in rare-earth-doped $\mathrm{CaFe}_{2} \mathrm{As}_{2}$ can be used to tune the superconducting state. Simultaneous changes in the 
superconductivity and magnetism in response to an applied electric current can be ascribed to the unstable Fe magnetic state or self-assembled superconductor/magnet composites in the $\mathrm{CaFe}_{2} \mathrm{As}_{2}$ compounds. These results provide a new route for investigating the role of $\mathrm{Fe}$ in $\mathrm{Fe}$ based superconductors. In addition, current-driven magnetization switching is expected to guide the design of new functional superconducting devices, such as superconductor-based on/off switch systems and superconducting spintronics ${ }^{37}$.

\section{Acknowledgements}

We thank S. Park, J. H. Yun, and T. H. Park for helpful discussions. This work was supported by the National Research Foundation (NRF) of Korea grant funded by the Korean Ministry of Science, ICT and Planning (No. 2012R1A3A2048816). S.-G.J. was also supported by Basic Science Research Program through the National Research Foundation of Korea (NRF) funded by the Ministry of Education (NRF-2015R1D1A1A01060382). This work is partly supported by a Grant-in-Aid for Scientific Research from the Ministry of Education, Culture, Sports, Science and Technology, Japan.

\section{Author details \\ ${ }^{1}$ Center for Quantum Materials and Superconductivity (CQMS), Department of Physics, Sungkyunkwan University, Suwon 16419, Republic of Korea. ${ }^{2}$ Department of Physics, Sungkyunkwan University, Suwon 16419, Republic of Korea. ${ }^{3}$ Department of Materials Science and Engineering, Seoul National University of Science and Technology, Seoul 01811, Republic of Korea. ${ }^{4}$ Department of Applied Physics, The University of Tokyo, Bunkyo-ku, Tokyo 113-8656, Japan}

\section{Conflict of interest}

The authors declare that they have no conflict of interest.

\section{Publisher's note}

Springer Nature remains neutral with regard to jurisdictional claims in published maps and institutional affiliations.

Supplementary information is available for this paper at https://doi.org/ 10.1038/s41427-018-0030-9.

Received: 20 October 2017 Revised: 2 February 2018 Accepted: 5 February 2018.

Published online: 11 April 2018

\section{References}

1. Yildirim, T. Strong coupling of the Fe-spin state and the As-As hybridization in iron-pnictide superconductors from first-principle calculations. Phys. Rev. Lett. 102, 037003 (2009).

2. Ortenzi, L. et al. Structural origin of the anomalous temperature dependence of the local magnetic moments in the $\mathrm{CaFe}_{2} \mathrm{As}_{2}$ family of materials. Phys. Rev. Lett. 114, 047001 (2015).

3. Stavrou, E. et al. Formation of As-As interlayer bonding in the collapsed tetragonal phase of $\mathrm{NaFe}_{2} \mathrm{As}_{2}$ under pressure. Sci. Rep. 5, 9868 (2015).

4. Gretarsson, H. et al. Spin-state transition in the Fe pnictides. Phys. Rev. Lett. 110, 047003 (2013).

5. Furukawa, Y., Roy, B., Ran, S., Bud'ko, S. L. \& Canfield, P. C. Suppression of electron correlations in the collapsed tetragonal phase of $\mathrm{CaFe}_{2} \mathrm{As}_{2}$ under ambient pressure demonstrated by ${ }^{75}$ As NMR/NQR measurements. Phys. Rev. B 89, 121109(R) (2014).

6. Saparov, B. et al. Complex structures of different $\mathrm{CaFe}_{2} \mathrm{As}_{2}$ samples. Sci. Rep. $\mathbf{4}$, 4120 (2014).

7. Kreyssig, A. et al. Pressure-induced volume-collapsed tetragonal phase of $\mathrm{CaFe}_{2} \mathrm{As}_{2}$ as seen via neutron scattering. Phys. Rev. B 78, 184517 (2008).

8. Basov, D. N. \& Chubukov, A. V. Manifesto for a higher Tc. Nat. Phys. 7, 272-276 (2011).
9. Park, T. et al. Hidden magnetism and quantum criticality in the heavy fermion superconductor CeRhln ${ }_{5}$. Nature 440, 65-68 (2006).

10. LV , B. et al. Unusual superconducting state at $49 \mathrm{~K}$ in electron-doped $\mathrm{CaFe}_{2} \mathrm{As}_{2}$ at ambient pressure. Proc. Natl Acad. Sci. USA 108, 15705-15709 (2011).

11. Gofryk, K. et al. Local inhomogeneity and filamentary superconductivity in PrDoped $\mathrm{CaFe}_{2} \mathrm{As}_{2}$. Phys. Rev. Lett. 112, 047005 (2014).

12. Saha, S. R. et al. Structural collapse and superconductivity in rare-earth-doped $\mathrm{CaFe}_{2} \mathrm{As}_{2}$. Phys. Rev. B 85, 024525 (2012).

13. Deng, L. Z. et al. Evidence for defect-induced superconductivity up to $49 \mathrm{~K}$ in $\left(\mathrm{Ca}_{1-x} R_{x}\right) \mathrm{Fe}_{2} \mathrm{As}_{2}$. Phys. Rev. B 93, 054513 (2016).

14. Saha, S. R. et al. Segregation of antiferromagnetism and high-temperature superconductivity in $\mathrm{Ca}_{1-x} \mathrm{La}_{x} \mathrm{Fe}_{2} \mathrm{As}_{2}$. Phys. Rev. B 89, 134516 (2014).

15. Korshunov, M. M., Efremov, D. V., Golubov, A. A. \& Dolgov, O. V. Unexpected impact of magnetic disorder on multiband superconductivity. Phys. Rev. B 90, 134517 (2014).

16. Bauer, E. D. et al. Electronic inhomogeneity in a Kondo lattice. Proc. Natl Acad. Sci. USA 108, 6857-6861 (2011).

17. Chudnovsky, E. M. Manipulating magnetic moments by superconducting currents. Phys. Rev. B 95, 100503(R) (2017).

18. Pershoguba, S. S., Björnson, K. Black-Schaffer, A. M. \& Balatsky, A. V. Currents induced by magnetic impurities in superconductors with spin-orbit coupling. Phys. Rev. Lett. 115, 116602 (2015).

19. Gao, Z. et al. Synthesis and properties of La-doped $\mathrm{CaFe}_{2} \mathrm{As}_{2}$ single crystals with $T_{c}=42.7 \mathrm{~K}$. EPL 95, 67002 (2011).

20. Lee, K-W., Pardo, V. \& Pickett, W. E. Magnetism driven by anion vacancies in superconducting a-FeSe ${ }_{1-x}$. Phys. Rev. B 78, 174502 (2008).

21. Choi, S. et al. Switching magnetism and superconductivity with spin-polarized current in iron-based superconductor. Phys. Rev. Lett. 119, 227001 (2017).

22. Sun, Y. et al. Evidence of two superconducting phases in $\mathrm{Ca}_{1-x} \mathrm{~L}_{x} \mathrm{Fe}_{2} \mathrm{As}_{2}$. AIP Adv. 3, 102120 (2013).

23. Tamegai, T., Ding, Q. P., Ishibashi, T. \& Nakajima, Y. Superconducting properties of $\mathrm{Ca}_{1-x} R E_{X} \mathrm{Fe}_{2} \mathrm{As}_{2}$ (RE: Rare Earths). Physica C 484, 31-34 (2013).

24. Zhao, K., Stingl, C., Manna, R. S., Jin, C. Q. \& Gegenwart, P. Reversible tuning of the collapsed tetragonal phase transition in $\mathrm{CaFe}_{2} \mathrm{As}_{2}$ by separate control of chemical pressure and electron doping. Phys. Rev. B 92, 235132 (2015).

25. Lee, S. F., Liou, Y., Yao, Y. D., Shih, W. T. \& Yu, C. Magnetic flux penetration depth study in Nb/Co system. J. Appl. Phys. 87, 5564-5566 (2000).

26. Kobayashi, S., Oike, H., Takeda, M. \& Itoh, F. Central peak position in magnetization hysteresis loops of ferromagnet/superconductor/ferromagnet trilayered films. Phys. Rev. B 66, 214520 (2002).

27. Roozmeh, S. E., Mohseni, S. M. \& Tehranchi, M. M. Study of magnetoimpedance effect of Co-based amorphous ribbons after current annealing at various kinds of ambient pressure. J. Non-Cryst. Solids 355, 2653-2656 (2009).

28. Muraca, R. D., Cremaschi, V., Knobel, M. \& Sirkin, H. Influence of Ge on magnetic and structural properties of Joule-heated Co-based ribbons: giant magnetoimpedance response. J. Magn. Magn. Mater. 320, 2068-2073 (2008).

29. Gomonay, H. V. \& Loktev, V. M. Spin transfer and current-induced switching in antiferromagnets. Phys. Rev. B 81, 144427 (2010).

30. Özyilmaz, B. et al. Current-induced magnetization reversal in high magnetic fields in Co/Cu/Co nanopillars. Phys. Rev. Lett. 91, 067203 (2003).

31. Prozorov, R. \& Kogan, V. G. London penetration depth in iron-based superconductors. Rep. Prog. Phys. 74, 124505 (2011).

32. Zhao, $\mathrm{K}$. et al. Interface-induced superconductivity at $\sim 25 \mathrm{~K}$ at ambient pressure in undoped $\mathrm{CaFe}_{2} \mathrm{As}_{2}$ single crystals. Proc. Natl Acad. Sci. USA 113, 12968-12973 (2016).

33. Xiao, $\mathrm{H}$. et al. Evidence for filamentary superconductivity nucleated at antiphase domain walls in antiferromagnetic $\mathrm{CaFe}_{2} \mathrm{As}_{2}$. Phys. Rev. B 85, 024530 (2012).

34. Cui, J. et al. Coexistence of antiferromagnetic and ferromagnetic spin correlations in $\mathrm{Ca}\left(\mathrm{Fe}_{1-x}\left(\mathrm{CO}_{x}\right)_{2} \mathrm{As}_{2}\right.$ revealed by ${ }^{75} \mathrm{As}$ nuclear magnetic resonance. Phys. Rev. B 94, 174512 (2016).

35. Wei, $F$. et al. The unusually high- $T_{C}$ in rare-earth-doped single crystalline $\mathrm{CaFe}_{2} \mathrm{As}_{2}$. Philos. Mag. 94, 2562-2569 (2014)

36. Zhou, W. et al. Anomalous electron doping independent two-dimensional superconductivity. New J. Phys. 19, 073014 (2017).

37. Linder, J. \& Robinson, J. W. A. Superconducting spintronics. Nat. Phys. 11, 307-315 (2015). 